\title{
Elevation of Cardiac Troponins in Prolonged Status Epilepticus: A Retrospective Chart Analysis
}

\author{
NG Soundarya ${ }^{1 *}$, DM Lawrence ${ }^{1}$, JB Samip ${ }^{1}$, AV Stacy ${ }^{2}$, JC Robert ${ }^{2}$, RT Leroy ${ }^{2}$ and RT Alan ${ }^{1}$ \\ ${ }^{1}$ Department of Neurology, Virginia Commonwealth University, USA \\ ${ }^{2}$ Department of Biostatistics, Virginia Commonwealth University, USA
}

Received: February 05, 2014; Accepted: March 14, 2014; Published: March 17, 2014

*Corresponding author: Soundarya N Gowda, Assistant Professor of Neurology, Associate Program Director and Medical Director of Epilepsy Monitoring Unit, Virginia Commonwealth University, 417N, 11th street, Po box: 980599, Richmond VA 23233, USA, Tel: 804-828-4323; Fax: 804-828-3667; E-mail:SNGowda@mcvh-vcu.edu

\begin{abstract}
Introduction: To determine the clinical significance of elevation of Troponin-I [cTn-I] during prolonged status_epilepticus [pSE] SE is known to be accompanied by an increase in sympathetic outflow. Elevation of cTn-I has been linked to myocardial stress. We hypothesize that in patients with risk factors for coronary artery disease[CAD], pSE may lead to myocardial stress and an elevation of cTn-I.
\end{abstract}

Methods: This is a retrospective study of patients over the age of 18 years who were presented to Virginia Commonwealth University with SE between 2005 and 2010. Data was evaluated using the 30-minute definition for SE and 30 day mortality. Risk factors for CAD and cTn-I levels within the first 24 hours of diagnosis of pSE were analyzed.

Key findings: There were a total of 435 patients with a confirmed diagnosis of pSE, of which 266 had cTn-I concentrations reported. Statistical analysis showed a significant association between CAD risk factors and cTn-I elevation ( $\chi 2=12.87$, p-value $<0.01$ ), with Crude Odds Ratio of 4.7. In patients with a CAD risk factor, an elevation of cTn-I is associated with a significantly increased risk of mortality, with an Odds ratio of $8.0,(\chi 2=40$, [95\% CI 4.1-15.9] p-value $<0.01)$. Mortality was higher in those with an elevation of cTn-I [54.65\%] as opposed to those who did not have an elevation [15.08\%], irrespective of CAD risk factors. $\mathrm{OR}=6.7,(\chi 2=45,[95 \% \mathrm{CI}=3.7-12.2] \mathrm{p}$-value $<0.01)$.

Conclusions: In patients with pSE values, elevated cTn-I values are seen four to five time more often in those with CAD risk factors, as opposed to those without the risks. An elevation of cTn-I in this subgroup of patients with CAD risk factors was associated with an eight to nine fold increase in their 30 day mortality as compared to patients with pSE, who did not have an elevation of cTn-I.

Keywords: Prolonged status epilepticus; Cardiac injury; Mortality

\section{Introduction}

Status epilepticus (SE) as defined for most research studies is continuous or repetitive seizures without intervening recovery of consciousness for 30 minutes or more [1]. Current definitions of SE for clinical purposes is, seizures lasting 10 minutes or more or two or more seizures in that time frame without return to baseline. It is a neurological and medical emergency and is associated with mortality rate as high as $22 \%$ [2]. Prior studies have shown that mortality is higher in the neonates and elderly, those receiving mechanical ventilation, patients with hypoxic ischemic brain injury and cerebrovascular diseases [3]. In the case of non-convulsive SE, mortality was higher in patients who had SE secondary to underlying medical conditions, those with severe impairment of mental status and patients with acute complications [4]. Mortality associated with SE is attributed to acute hypertension and tachycardia leading to subsequent development of pulmonary edema, hypotension, cardiac arrhythmias and circulatory collapse [5-7]. Animal studies and case reports suggest a chronic alteration in autonomic regulation of cardiac function, characterized by increased sympathetic dominance of the vagal system as an underlying mechanism for cardiac arrhythmias and myocardial damage in form of contraction band necrosis, myocytolysis and Takotsubo cardiomyopathy in the setting of SE [8-10].

Troponins complex consists of three subunits- Troponin-C, Troponin-I and Troponin-T. These are located on the actin filament of striated muscles (Cardiac and Skeletal). Troponin-T and Troponin-I are only expressed in cardiac muscles and in the year 2000, the European Society of Cardiology and the American College of Cardiology committee jointly redefined myocardial infarction (MI) by an elevation of cardiac Troponin-T (cTn-T) or Troponin-I (cTn-I) in conjunction with clinical evidence of myocardial ischemia [11]. It is also known that elevation of cTn-T or cTn-I is seen in patients without acute coronary syndromes in conditions such as myocarditis, pulmonary embolism, acute and chronic heart failure, septic shock, use of cardio toxic drugs, and strenuous exercise [12-14]. There have been reports of spurious elevations of cTn-T along with myoglobin in patients with diabetes as well as chronic kidney disease. $[15,16]$. There is evidence to prove that elevation of cTn-I accurately predicts myocardial injury even in patients with renal failure [17].

Currently, published data regarding significance of elevated troponin in patients with prolonged status epilepticus [pSE] is limited. We hypothesized that pSE leading to sympathetic 
overflow, in patients with risk for coronary artery disease (CAD) causes myocardial stress. This in turn leads to an elevation of cTn-I and cTn-T due to myofibrillar injury. An increase in mortality among those patients with SE who have an elevation of cTn-I or T will corroborate this hypothesis. The purpose of this study is to find if a correlation exists between elevation of cTn-I and presence of CAD risk factors in patients with SE and to determine if this elevation of cTn-I would be associated with an increase in mortality.

\section{Materials and Methods}

This is a retrospective study on all patients over the age of 18 years who presented to Virginia Commonwealth University with status epilepticus between 2005 and 2010. Data from the Greater Richmond Metropolitan Area Status Epilepticus Project (GRMASE) database were evaluated using the 30-minute definition for SE. This study was conducted with our institutional IRB approval [IRB number: HM10406] and patient information was de-identified. Both convulsive and non-convulsive seizures were included in the analysis. Age, risk factors for CAD (as per current National Heart, Lung and Blood Institute guidelines) and cTn-I levels during the period of SE or within the first 24 hours of diagnosis were collected. The number of risk factors was not quantified since this information may not always be accurately known at the time of presentation. Mortality data was collected at 30 days after cessation of SE.

The risk factors for CAD as described by the National heart, Lung and Blood Institute guidelines are dyslipidemia, high blood pressure, smoking, insulin resistance, diabetes, obesity, metabolic syndrome, lack of physical activity, unhealthy diet, family history of early heart disease and older age (men greater than 45 and women greater than 35).

There were a total of 435 patients with a confirmed diagnosis of SE at this institution in the above mentioned time period. cTn-I concentrations were measured at the time of admission or within 24 hours of onset of seizures in 266 of the 435 patients. A numerical cutoff value for enzyme elevation was not used for analysis of this study and data was gathered primarily as either within normal range or elevated. This had to be done since the normative values had changed along the time span of this study due to changes in the reagents and reporting values used by the laboratory and there is no way to correlate results from a Troponin-I assay to Troponin-T assay and often even between Troponin-I assays themselves. Therefore for each of the assay used, we collected data as either "elevated" or "normal" based on the upper limit of numerical cutoff value reported for the particular assay used at the time. Patients were excluded if they either had a concurrent myocardial infarction or their SE was secondary to anoxic brain injury due to cardiopulmonary arrest. This subset of thirty one patients were excluded since the elevation of cTn-I could be a direct result of the resuscitation, cardiac arrest and also because pSE in this population is traditionally associated with higher mortality rates. Of the data available for analysis with regards to etiology of pSE, 108 patients had pSE without any underlying co morbidities, 12 patients had pSE secondary to cerebrovascular accidents and 57 patients had pSE due to an acute symptomatic etiology such as underlying medical conditions, intracranial hemorrhage, intoxication, mass lesions and others. The data on remaining patients were either unavailable or unclear.

Chi-squared tests were performed to test the univariate association of cTn-I concentrations with CAD risk factor (yes or no). All statistical analyses were performed using SAS 9.3 (SAS Institute Inc., Cary, $N C$, USA).

\section{Results and Discussion}

Statistical analysis showed a significant association between CAD risk factors and cTn-I elevation $(\chi 2=12.87$, p-value $<0.01)$. Fisher's exact test was performed due to the small sample size of one cell and it verified the results $(\mathrm{p}<0.01)$. The Crude Odds Ratio for CAD risk factor is 4.7 (95\% Confidence Interval $[1.9,11.5]$ ), denoting the odds for a troponin elevation in a patient with a CAD risk factor were 4.7 times higher than the odds for a troponin elevation in the absence of a CAD risk factor.

See Table 1: Association between elevation of cTn-I and presence or absence of CAD risk factors.

This test also showed no significant association between age and cTn-I concentration $(\chi 2=1.80, p$-value $=0.1793)$. The Crude Odds Ratio was 1.5 (95\% C.I [0.8, 2.8]).

See Table 2: Association between age and elevation of cTn-I.

The results of the logistic regression model show that age alone is not a significant predictor of the probability of cTn-I elevation, but having a CAD risk factor is significantly associated with cTn-I elevation. That is, once adjusted for age, an individual with a CAD risk factor is 4.5 times as likely to have elevated cTn-I as is an individual in the same age group who does not have any CAD risk factors.

See Table 3: Logistic regression model of age grouping and cTn-I elevation.

Since age by itself was not found to be significant, the CAD risk factor was adjusted to include all age greater than 18 years, which was our target demographic. This relationship between CAD risk factor and cTn-I elevation was found to be statistically significant $(\chi 2=10.64, \mathrm{p}$-value $<0.01)$. With the presence of one small cell size, the results of this test match that of Fisher's exact test. The odds ratio for this table is 6.1 (95\% C.I. [1.8, 20.4]).

Table 1: Association between elevation of cTn-I and presence or absence of CAD risk factors.

\begin{tabular}{|l|c|c|c|}
\hline & cTn-I elevated & cTn-I normal & Total \\
\hline CAD risk present & $51^{*}(\mathrm{p}<0.01)$ & 115 & 166 \\
\hline CAD risk absent & 6 & 63 & 69 \\
\hline Total & 57 & 178 & 235 \\
\hline
\end{tabular}

Table 2: Association between age and elevation of cTn-I.

\begin{tabular}{|l|c|c|c|}
\hline & cTn-I elevated & cTn-I normal & Total \\
\hline Age over 60 years & 34 & 88 & 122 \\
\hline Age less than 60 yr & 23 & 90 & 113 \\
\hline Total & 57 & 178 & 235 \\
\hline
\end{tabular}


In patients with a CAD risk factor, an elevation of cTn-I is associated with a significantly increased risk of mortality with an odds ratio of 8.0, ( $\chi 2=40$, [95\% CI 4.1-15.9] p-value $<0.01)$. The same degree of association with increased mortality was not seen with relation to CAD risk factors alone. $(\chi 2=1.35$, [95\% CI 0.6-2.6] $\mathrm{p}$-value $=0.3$ ). Mortality was higher in those with an elevation of cTn-I_[54.65\%], irrespective of whether they did or did not have CAD risk factors. OR=6.7, $(\chi 2=45,[95 \% \mathrm{CI}=3.7-12.2] \mathrm{p}$-value < $0.01)$. See Table 4: Association between mortality and cTn-I and CAD risk.

Overall 30 day mortality was $7.4 \%$ in those with pSE in this database, $38 \%$ in those with acute symptomatic etiology and $9.09 \%$ in those with concurrent cerebrovascular accidents. Statistically significant correlation between elevated cTn-I and mortality was strongest in patients with cryptogenic pSE: OR 17 (95\%CI of 3.13-92, p value of 0.001). Those with acute symptomatic etiology for pSE and cerebrovascular accidents as cause of pSE did not show a similar correlation between elevated cTn-I and mortality on subgroup analysis. OR=4.15 (95\% CI 1.0915 , $p$ value of 0.03 ).

\section{Conclusion}

The most important findings from this study are that in patients with pSE an elevated CTn-I is seen four to five times more often than in those with CAD risk factors, as opposed to those without the risk factors. An elevation of cTn-I in this subgroup of patients with CAD risk factors was associated with an eight to nine fold increase in their 30 day mortality as compared to patients with pSE, who did not have an elevation of cTn-I. This trend was seen irrespective of the age of the patient. Assuming optimal treatment, it is uncommon for patients to die during SE and generally SE related mortality peaks within 30 days after SE [18]. The major risks for death associated with pSE are, myoclonic SE in post anoxic patients, SE lasting more than 24 hours, acute symptomatic etiologies, and age [19]. Cardiac arrhythmias, Takotsubo cardiomyopathy are other etiologies implicated towards increasing mortality. SE following post anoxic brain injury and post cardiopulmonary resuscitation is

Table 3: Logistic regression model of age grouping and cTn-I elevation.

\begin{tabular}{|l|c|c|c|c|c|c|}
\hline Parameter & Estimate & S.E. & Wald Chi-Sq & P-value & $\begin{array}{c}\text { Adj. } \\
\text { OR }\end{array}$ & $\begin{array}{c}\mathbf{9 5 \%} \\
\text { Wald C.I. }\end{array}$ \\
\hline Intercept & 0.77 & 0.21 & 13.51 & $<0.01$ & & \\
\hline Age & 0.12 & 0.32 & 0.14 & 0.71 & 1.1 & $(0.6,2.1)$ \\
\hline $\begin{array}{l}\text { CAD Risk } \\
\text { Factor }\end{array}$ & 1.50 & 0.47 & 10.26 & $<0.01$ & 4.5 & $(1.8,11.3)$ \\
\hline
\end{tabular}

Table 4: Association between mortality and CTn-I and CAD risk.

\begin{tabular}{|l|c|c|c|}
\hline & Alive & Dead & Total \\
\hline Elevated cTn-I with CAD & 37 & $44^{*}(\mathrm{p}<0.01)$ & 81 \\
\hline Elevated cTn-I without CAD & 2 & 3 & 5 \\
\hline Normal cTn-I with CAD & 108 & 16 & 124 \\
\hline Normal cTn-I without CAD & 44 & 11 & 55 \\
\hline
\end{tabular}

traditionally associated with higher mortality, and this subgroup of patients were therefore excluded from this analysis.

The findings of this study suggest that in patients with risk factors of CAD, SE may act as an acute stressor. We hypothesize that with pSE, there is an increase in sympathetic outflow leading to an increase in oxygen demand of myocardium due to resultant tachycardia and other effects of sympathovagal dominance. Catecholamine released directly from nerve terminals into the myocardium are suspected to be the culprits leading to contraction band necrosis and cardiac damage [20]. In the presence of risk factors for CAD, a mismatch in demand and supply is assumed under these situations, which results in injury to myocardium leading to elevation of cTn-I.

Since data was gathered prospectively in this study, and analyzed later, the implications of elevated CTn-I in patients with SE, without symptoms or other signs of acute coronary syndrome was not known at the time. This is one of the limitations of this study. There were two patients who also demonstrated abnormalities on electrocardiogram. One of these patients did undergo coronary angiogram; however no significant abnormality was documented. Other limitations include lack of a concomitant electrocardiography test in these patients, lack of confirmatory coronary angiographic or pathology results to confirm our hypothesis. Other variables that have not been addressed in this study are refractoriness to treatment and duration of pSE, pre-existing co-morbidities that could also contribute to increased mortality. The lack of our ability to test these variables and their correlation to CAD risk and cTn-I elevation and subsequent mortality does limit the strength of our postulated causal association. In future, further studies can be undertaken to address these individually. We were unable to ascertain the correlation between the duration of status and mortality, due to the limitations of recollection bias that came with the retrospective nature of our study. While the troponin levels were all checked and only considered if drawn within 24 hours of onset of SE, the exact duration of episode of SE was not documented. We regret the lack of inclusion of this important piece of information. The lack of a numerical value for cTn-I level is also a limitation, but there is no way to correlate results between various Troponin-I assays.

This study is one of the largest published series to date and the statistical significance of association between increased mortality in patients with SE who have CAD risk factors and elevated cTn-I is overwhelming. Further prospective-interventional studies are warranted to determine if we can alter mortality risk by addressing the cardiac risk factors concurrently while treating SE. This will require close collaboration with cardiology and intensive care.

\section{Acknowledgement}

The bio statistical analysis of the project described was supported by award number UL1RR031990 from the National Center for Research Resources.

We would like to thank all the Faculty and Study associates involved in the NIH Status Epilepticus project at VCU for their 
valuable contributions. The project described was supported by award number-5R01NS051505-05 from the National Institutes of Health.

\section{References}

1. DeLorenzo RJ, Garnett LK, Towne AR, Waterhouse EJ, Boggs JG, et al. (1999) Comparison of status epilepticus with prolonged seizure episodes lasting from 10 to 29 minutes. Epilepsia 40(2): 164-169.

2. DeLorenzo RJ, Hauser WA, Towne AR, Boggs JG, Pellock JM, et al. (1996) A prospective, population-based epidemiologic study of status epilepticus in Richmond, Virginia. Neurology 46(4): 1029-1035.

3. Koubeissi M, Alshekhlee A (2007) In-hospital mortality of generalized convulsive status epilepticus. A large US sample. Neurology 69(9): 886893.

4. Shneker BF, Fountain NB (2003) Assessment of acute morbidity and mortality in nonconvulsive status epilepticus. Neurology 61(8): 1066-1073.

5. Schraeder Pl, Lathers CM (1983) Cardiac neural discharge and epileptogenic activity in cat: an animal model for unexplained death. Life sci 32(12): 1371-1382.

6. Zweifler RM, Slaven EM, Rihn LL, Magee JC, Kreisman NR (1991) Renal hemodynamic changes during serial seizures in rats. Am J Physiol 261(5): H1508-1513.

7. Walton NY (1993) Systemic effects of generalized convulsive status epilepticus. Epilepsia 34(1): S54-S58.

8. Bealer SL, Little JG, Metcalf CS, Brewster AL, Anderson AE (2010) Autonomic and cellular mechanisms mediating detrimental cardiac effects of status epilepticus. Epilepsy Res 91(1): 66-73.

9. Metcalf CS, Radwanski PB, Bealer SL (2009) Status epilepticus produces chronic alterations in cardiac sympthovagal balance. Epilepsia 50(4): 747-754.

10. Legriel S, Bruneel F, Bedos JP, Appere-de-VC, Georges JL, et al. (2008)
Recurrent Takotsubo cardiomyopathy triggered by convulsive status epilepsticus. Neurocrit care 9(1): 118-121.

11. Alpert JS, Thygesen K, Antman E, Bassand JP (2000) Myocardial infarction redefined- a consensus document of the Joint European Society of Cardiology/American College of Cardiology committee for the redefinition of myocardial infarction. J Am Coll Cardiol 36(3): 959-969.

12. Hamm CW, Giannitsis E, Katus HA (2002) Cardiac troponin elevation in patients without acute coronary syndrome. Circulation 106: 2871-2872.

13. Kroff S, Katus HA, Giannitsis E (2006) Differential diagnosis of elevated troponins. Heart 92(7): 987-993.

14. Goldmann BU, Christenson RH, Christian WM, Thomas M, Ohman EM, et al. (2001) Implications of troponin testing in clinical medicine. Curr Control Trials Cardiovasc Med 2(2): 75-84.

15. Li D, Keffer J, Corry K, Vazquez M, Jailal I (1995) Nonspecific elevation of troponin $\mathrm{T}$ levels in patients with chronic renal failure. Clin Biochem 28(4): 474-477.

16. Haller C, Stevanovich A, Katus HA (1996) Are Cardiac troponins reliable serodiagnostic markers of cardiac ischemia in end-stage renal disease? Nephrol Dial Transplant 11: 941-944.

17. Martin GS, Becker BN, Schulman G (1998) Cardiac troponin-I accurately predicts myocardial injury in renal failure. Nephrol Dial Transplant 13(7): 1709-1712.

18. Boggs JG (2004) Mortality associated with Status Epilepticus. Epilepsy Curr 4(1): 25-27.

19. Logroscino G, Hesdorffer DC, Cascino GD, Annegers JF, Bagiella E, et al. (2002) Long-term mortality after first episode of status epilepticus. Neurology 58(4): 537-547.

20. Manno EM, Pfeifer EA, Cascino GD, Noe KH, Wijdicks EFM (2005) Cardiac pathology in Status Epilepticus. Ann Neurol 58(6): 954-957. 